\title{
BMJ Open Prevalence of psoriasis and associated risk factors in China: protocol of a nationwide, population-based, cross- sectional study
}

\author{
Jing Li, ${ }^{1}$ Meiwen Yu, ${ }^{1}$ Ya-wen Wang, ${ }^{\odot}$ Jia-an Zhang, ${ }^{1}$ Mei Ju, ${ }^{1}$ Kun Chen, ${ }^{1}$ \\ Yu Jiang, ${ }^{2}$ Min Li, ${ }^{1,3}$ Xiang-Sheng Chen ${ }^{1}$
}

To cite: Li J, Yu M, Wang Y, et al. Prevalence of psoriasis and associated risk factors in China: protocol of a nationwide, population-based, crosssectional study. BMJ Open 2019;9:e027685. doi:10.1136/ bmjopen-2018-027685

- Prepublication history for this paper is available online. To view these files, please visit the journal online (http://dx.doi org/10.1136/bmjopen-2018027685).

$\mathrm{JL}$ and MY contributed equally.

Received 2 November 2018

Revised 2 April 2019

Accepted 10 June 2019

Check for updates

(C) Author(s) (or their employer(s)) 2019. Re-use permitted under CC BY-NC. No commercial re-use. See rights and permissions. Published by BMJ.

1 Institute of Dermatology, Jiangsu Key Laboratory of Molecular Biology for Skin Diseases and STIs, Chinese Academy of Medical Sciences and Peking Union Medical College, Nanjing, China

${ }^{2}$ School of Public Health, Chinese Academy of Medical Sciences and Peking Union Medical College, Beijing, China

${ }^{3}$ Center for Global Health,

School of Public Health, Nanjing Medical University, Nanjing, China

Correspondence to

Dr Min Li;

limin@pumcderm.cams.cn

\section{ABSTRACT}

Introduction Psoriasis is a chronic inflammatory skin disease which could lead to serious complications and increased risk of cardiovascular diseases. Psoriasis was recognised as a serious non-communicable disease with important public health impact by member states in the World Health Assembly resolution in 2014. However, data on psoriasis epidemiology are scarce worldwide, especially from low-income and middle-income countries. Only a few epidemiological studies on psoriasis have been conducted in parts of China, mostly without appropriate sampling design and data analysis.

Aim This study aims to obtain the prevalence of psoriasis in China and relevant risk factors through a nationwide, population-based study with adequate statistical design. Methods and analysis This is a cross-sectional study to be conducted in 60 sites across China. A multistage, cluster random sampling design is used. Participants should have local household registration or be residing in the survey area for at least 6 months during the past year. The presence of psoriasis is ascertained independently by two certified dermatologists. If any discrepancies in the diagnosis occur, consensus will be met via discussion. All participants will be interviewed with a questionnaire to collect sociodemographic and disease information. The field survey will be implemented from October 2018 to June 2019. All statistical analyses will be conducted using survey procedures in SAS V.9.2 software to adjust for the complex sample design.

Ethics and dissemination The study has been reviewed and approved by the ethics committee of the Institute of Dermatology, Chinese Academy of Medical Sciences and Peking Union Medical College (Nanjing, China). A written informed consent will be obtained from all participants before the questionnaire survey. Findings of the study will be disseminated through publications in peer-reviewed journals.

\section{BACKGROUND}

Psoriasis is a common, chronic and immune-mediated inflammatory skin disease with no clear aetiology. ${ }^{1}$ It affects men and women of all ages across the world. In addition to the involvement of the skin and nails,
Strengths and limitations of this study

- The study will provide data on the prevalence of psoriasis and related risk factors among the Chinese population, which will be useful for estimating disease burden and healthcare planning.

- A national, population-based, cross-sectional study with appropriate sampling design will be conducted.

- Psoriasis cases will be ascertained and evaluated by two trained dermatologists independently rather than via patient report to ensure accurate case classification.

- The rural sample may miss a significant proportion of adults due to population migration, which may potentially affect the representativeness of the study.

psoriatic arthritis, which leads to joint deformations and disability, may develop. ${ }^{1}$ Moreover, patients with psoriasis are reported to be at increased risk of developing cardiovascular and other non-communicable diseases (NCDs).$^{2-5}$ Individuals with psoriasis have reduced quality of life, suffer from depression and experience significant social stigma. ${ }^{6-8}$

The reported prevalence of psoriasis varied from $0.09 \%$ in Tanzania to $11.4 \%$ in Norway, making psoriasis a serious global problem with at least 100 million people affected worldwide. ${ }^{9}$ Psoriasis appears to be most prevalent in Northern Europe and least in Eastern Asia. ${ }^{9} 10$ Studies in several countries suggest that the prevalence of psoriasis may be increasing. ${ }^{9} 10$ In 2014, psoriasis was recognised as a serious NCD by member states in the World Health Assembly resolution (WHA67.9). ${ }^{11}$ However, available data on the prevalence and incidence of psoriasis are mostly from the USA and European countries. ${ }^{12}$ There is a big gap in the availability of epidemiological data on psoriasis worldwide, especially from low-income and middle-income countries. WHO published a report to 
draw attention to the public health impact of psoriasis. ${ }^{11}$ The report highlights that a key area of research globally is to obtain quality data on the epidemiology of psoriasis to better understand the size and distribution of the problem. Such data are essential for disease control and appropriate healthcare planning.

In mainland China, a nationwide survey in 1984 revealed that the prevalence of psoriasis was $0.16 \%$, meaning that there could be about 2 million patients with psoriasis in China given its big population size (1.33 billion in 2010 census). ${ }^{13}$ Since then, some epidemiological studies have been conducted in single cities or regions, mostly without statistically appropriate sampling design and data analysis. ${ }^{14-18}$ The most recent study was conducted in purposively selected six cities in 2008 and found that the prevalence of psoriasis was $0.59 \%$, indicating a slight increase compared with the prevalence in $1984 .{ }^{17}$ However, these studies showed inconsistent findings in gender and geographical differences in the prevalence of psoriasis, which were probably due to differences in study population, geographical location and case ascertainment methods. Therefore, this study aims to obtain the prevalence of psoriasis in China and relevant risk factors through a nationwide, population-based study with adequate statistical design.

\section{METHODS}

\section{Study population}

The target population of the study is non-institutionalised Chinese nationalities living in mainland China, consisting of 31 provinces, municipalities and autonomous regions (hereafter all termed as provinces). To be eligible for the study, individuals should have local household registration or be residing in the survey area for at least 6 months prior to survey implementation. No age limit is applied.

\section{Sample size calculation}

Since the prevalence of psoriasis is very low, the following formula is used to calculate sample size. ${ }^{19}$ The national prevalence of psoriasis is assumed to be $0.2 \%$ and the relative error is set to be 0.25 .

$$
\mathrm{n}=\left[\frac{57.3 \mathrm{u}_{\frac{\alpha}{2}}}{\sin ^{-1}[\delta / \sqrt{\pi(1-\pi)}]}\right]^{2}
$$

where $\Phi$ is the prior guess of the national prevalence of psoriasis, that is, $0.2 \% ; \delta$ is the relative error which is set to be 0.25 ; and $\alpha$ is type I error, that is, 0.05 . When $\varpi$ is very low, arcsine transformation is used to approximate the normal distribution for calculating the sample size.

Due to a cluster sample survey design, the sample size must be increased by the multiple of the so-called 'design effect' relative to the sample size required for a simple random sample. ${ }^{2021}$ This is to account for the fact that less information is provided due to inherent interdependence between individuals within the same cluster than simple random sampling. A design effect of 1.5, which has been used in other studies with similar complex sampling design and cluster size, is used..$^{21}$ The final step in the sample size calculation is to increase the sample size to allow for non-participation in the study. ${ }^{21}$ After adjusting for a preassumed participation rate of $80 \%$, the total sample size required is estimated to be 57515 , which is rounded to 60000 for convenience of sample allocation.

\section{Sampling design and procedure}

A multistage, cluster random sampling design is applied. ${ }^{20}$ A cluster size of 1000 eligible individuals is chosen mainly to maximise the probability of finding psoriasis cases at each site and complete data collection for a cluster within a convenient time period (like 1 or 2 weeks). ${ }^{21}$ Thus, a total of 60 clusters are to be selected with an overall ratio of urban to rural sites of 29 to 31, in line with the urban to rural ratio in the 2010 national census. A cluster is defined as a group of households with at least 1000 eligible individuals residing in a community within a district/town, or in a village within a township.

For ease of organising survey implementation, the hierarchical administrative division of China is used as sampling units. The number of clusters allocated to each province is proportional to the population size of that province. In the first step, 60 administrative units (urban districts or counties) are selected, stratified by provinces with probability proportional to population size. In the second stage, one residential community within the selected district or one administrative village within the selected county will be selected with probability proportional to population size. In the final stage, within the selected residential community or administrative village, a list of residential clusters consisting of 1250 residents to allow for non-participation will be compiled using official community or village registers of households and migrants. Then one cluster will be randomly selected from the list. All individuals within the selected cluster will be screened for eligibility and invited to participate in the study. If the number of eligible individuals in a cluster is fewer than 1000 people, then persons from the next neighbouring cluster will be recruited to reach the target size.

\section{Case ascertainment}

Psoriasis is characterised by red patches covered with silvery-white scabs of dead skin. The presence of psoriasis will be determined independently by two certified dermatologists. A discussion will be needed to resolve any discrepancies in the diagnosis and to reach consensus.

\section{Survey implementation}

The field survey will be implemented from October 2018 until June 2019. Respondents will be invited to a gathering point that is easy to get to and safe to participate in the study. For certain rural areas where it is not easy to get to a central point, house-to-house visits will be conducted. After obtaining informed consent, all participants will be interviewed by trained interviewers to collect 
information on demographic characteristics, personal and family medical history, and lifestyle risk factors. The demographic variables to be collected include age, gender, marital status, ethnicity, occupation and household registration. Lifestyle risk factors include alcohol and tobacco use. All participants will undergo physical measurements of height, weight, waist circumference and blood pressure according to a standard protocol. Participants reporting any skin manifestations will be evaluated by dermatologists to assess whether they have psoriasis. Those who meet the case definition will continue the questionnaire survey to collect information on disease characteristics, including onset sites, onset age, causes of relapse or flare episodes, joint symptoms, concomitant conditions, and treatment history during the course of the disease. The clinical types of psoriasis will be determined. Medical history of hypertension, hyperlipidaemia, diabetes and coronary heart disease that have been diagnosed by physicians will also be enquired. Body surface area and the Psoriasis Area Severity Index will be used to evaluate disease severity. All interviews will be assisted by a smartphone application with computerised questionnaires incorporating conditional skips and logic checks.

Individuals with psoriasis will be offered tests for alanine aminotransferase (ALT), aspartate aminotransferase (AST), blood urea nitrogen, serum creatinine, cholesterol, triglycerides and fasting food glucose.

\section{Risk of bias}

A rigorous plan will be followed to reduce the risk of bias and ensure the validity of the study. To reduce the risk of misclassification, potential cases are to be confirmed independently by two certified dermatologists. Any discrepancies will be resolved through discussion or by a third dermatologist. It is expected that some individuals will not be able to or not volunteer to participate. It could affect the validity of the study when a high proportion of individuals choose not to participate in the study. Several measures will be taken to encourage participation, including motivating key individuals at local communities such as directors of neighbourhood or village committees, publicising the study and mobilising local residents before starting the fieldwork. A particular concern lies in reaching migrant workers (the so-called floating population). Due to rapid urbanisation and industrialisation over the past four decades in China, tens of thousands of rural young labourers have moved to thriving coastal cities and concentrated industrial zones for better employment opportunities. ${ }^{23}$ According to the National Bureau of Statistics of China, it was estimated that there were 245 million rural-to-urban migrant workers in 2016. ${ }^{24}$ Although a temporary residence permit is required by local authority, many migrants do not obtain the permit and thus are not included in the registers of migrants. Since they are not at home most of the year and often not registered in cities, these migrant workers may be missed in rural areas and may not be well captured in the survey in urban areas receiving migrants. This phenomenon can potentially affect the representativeness of the study for rural areas, as well as urban areas receiving migrants if the prevalence of psoriasis is different between migrants with or without residence permit. Several measures will be taken to maximise the capture of migrant workers in the study, such as trying to enlist migrant workers within urban areas, conducting the interview through cellphone and confirming cases through pictures or video chats when someone is absent.

\section{Quality control and data management}

A series of stringent quality control measures will be taken to ensure the validity and reliability of study data. Before field implementation, questionnaires will be pretested and revised accordingly. Study staff at local sites will be trained on interview skills and the use of standardised protocols and questionnaires for data collection. Each questionnaire should be checked for completeness and errors before finishing interviews. Two certified dermatologists will be trained on the case definitions of psoriasis for the study. All participants will be evaluated for psoriasis independently by two dermatologists. Designated staff will conduct independent site monitoring for external quality assessment at regular intervals, according to the procedures and requirements of the study protocol.

Data collected locally will be sent electronically via the internet on a weekly basis. All paper-based data will be double-entered using the EpiData software (V.3.1, EpiData Association, Denmark) by two research assistants and compared for consistency to ratify data entry. Any discrepancies between the two data sets will be resolved prior to data analysis. All stored data will be non-identifiable, whereby all participants will be anonymous. Paper documents will be kept in a locked drawer of a filing cabinet at the research site. Electronic data will be stored on password-protected computer files which can only be accessible to the members of the research team.

\section{Statistical analysis}

Since a self-weighting design is used in the study, the design weight is calculated by multiplying the inverse of inclusion probabilities of sampling units in each stage. It needs to be adjusted to account for non-response and any structural deviation of the sample from the target population caused by the implementation process. Adjustment coefficient for non-response is calculated using non-response rate. Structural adjustment coefficient is calculated using the deviation in the distribution of certain key variables, such as age or gender, in the final sample from the target population (the 2010 China Population Census data). The final weight is to multiply the design weight by these adjustment coefficients.

To allow inference to the general population, all statistical analyses will be performed using survey procedures (PROC SURVEYFREQ, PROC SURVEYMEANS, PROC SURVEYREG, PROC SURVEYLOGISTIC) in SAS software (V.9.2) to incorporate sample weights and stratification by province of the complex sample design. Prevalence 
and covariate data will be reported as percentages with 95\% CI. Standardised prevalence of psoriasis according to various background characteristics including region, urban/rural place of residence and sociodemographic factors will be estimated using China's 2010 census data. Weighted ORs with $95 \%$ CI will be computed with survey logistic regression for both unadjusted and adjusted (for age, gender and other factors) analyses to examine the association of demographic and lifestyle factors with the odds of having psoriasis. Multilevel models will be used for data with hierarchical structure to accommodate the interdependence of subjects within the same cluster. Multiple imputations will be used to handle missing data. Data collected from confirmed psoriasis cases will be analysed to learn disease onset and other characteristics. A two-sided $p$ value less than 0.05 is considered statistically significant. All data will be presented in tables, charts and/or graphs.

\section{Ethical considerations}

Written informed consent will be obtained from all participants, or legal guardians of participants if necessary. Findings of the study will be disseminated as peer-reviewed publications in journals and will be presented at international and national conferences.

\section{Patient and public involvement}

The aim to understand the size of psoriasis burden and associated factors was partly informed by patients' enquires about how common psoriasis was and why they had psoriasis. Patients or the public were not involved in the design of the study. They will not be involved in the recruitment and implementation of the study. Newsletters will be sent to local community leaders to help circulate study results among participants. Media releases will be considered to increase public awareness of psoriasis.

\section{DISCUSSION}

This protocol outlines the rationale and design of a nationwide, population-based, cross-sectional study that aims to determine the prevalence of psoriasis and associated factors in mainland China. Only a few studies have looked at the prevalence of psoriasis in China, mostly in single cities or regions, with inconsistent findings. ${ }^{14-18}$ Given the large population and vast geographical diversity in China, it is of great importance to improve knowledge about psoriasis epidemiology. Better understanding of the size and distribution of the problem will be very useful for healthcare planning and disease control. ${ }^{11}{ }^{12}$ Our study is also a proactive response to the WHO's call to collect prevalence and incidence data on psoriasis for better estimation of the disease burden worldwide. It is expected that our effort of determining the prevalence of psoriasis in China will make important contribution to the initiative of Global Psoriasis Atlas. ${ }^{12}$

The strengths of the study include the diagnosis of psoriasis by trained dermatologists rather than patient report to ensure accurate case classification and a nationally representative sample to obtain estimates of national prevalence. In addition, appropriate sampling procedure and data analysis plan guided by statisticians can ensure the representativeness of the study. The large sample size allows for a comprehensive investigation of factors associated with having psoriasis.

There may be some limitations. As mentioned above, migrant workers are not at their rural home most of the year and often not registered in urban areas. Chances are high that these migrant workers may be missed in our study. Subsequently, the sample recruited from rural areas may miss a significant proportion of adults aged between 16 and 60. This phenomenon can potentially undermine the representativeness of the study for rural areas. On the other hand, migrants without residence permit may be missed in urban areas receiving migrants. If the prevalence of psoriasis is significantly different among migrants with or without residence permits, the bias due to rural-urban migration can also affect the areas receiving migrants. A small survey can be conducted to see whether having psoriasis is associated with having residence permits. We will apply several measures to maximise the capture of migrant workers in the study, such as trying to enlist migrant workers within urban areas, conducting the interview through cellphone and confirming cases through pictures or video chats when someone is absent. Standardised prevalence will be calculated to adjust for differences in age distribution when analysing data.

In summary, the problem of psoriasis in China has not received much attention despite its huge disease burden. Improved knowledge on the prevalence of psoriasis and associated factors has the potential to influence health resources allocation and provide insights for further research to better address this disabling systemic disease.

Contributors ML and X-SC conceptualised the study and secured funding. ML, $\mathrm{JL}, \mathrm{MY}, \mathrm{JZ}, \mathrm{MJ}$ and $\mathrm{KC}$ were involved in the design of the study. JL, YW and YJ formulated the sampling procedure and data analysis plan. JL and MY drafted the manuscript. All authors have approved the final version.

Funding This work is supported by CAMS Innovation Fund for Medical Sciences (2017-I2M-1-017).

Competing interests None declared.

Patient consent for publication Not required.

Ethics approval The study protocol has been reviewed and approved by the ethics committee of the Institute of Dermatology, Chinese Academy of Medical Sciences and Peking Union Medical College (Nanjing, China).

Provenance and peer review Not commissioned; externally peer reviewed.

Open access This is an open access article distributed in accordance with the Creative Commons Attribution Non Commercial (CC BY-NC 4.0) license, which permits others to distribute, remix, adapt, build upon this work non-commercially, and license their derivative works on different terms, provided the original work is properly cited, appropriate credit is given, any changes made indicated, and the use is non-commercial. See: http://creativecommons.org/licenses/by-nc/4.0/.

\section{REFERENCES}

1. Boehncke WH, Schön MP. Psoriasis. Lancet 2015;386:983-94.

2. Wan MT, Shin DB, Hubbard RA, et al. Psoriasis and the risk of diabetes: a prospective population-based cohort study. J Am Acad Dermatol 2018;78:315-22. 
3. Gelfand JM, Wan MT. Psoriasis: a novel risk factor for type 2 diabetes. Lancet Diabetes Endocrinol 2018;6:919-21.

4. Cozzani E, Rosa GM, Burlando M, et al. Psoriasis as a cardiovascular risk factor: updates and algorithmic approach. G Ital Dermatol Venereol 2018;153.

5. Charlton R, Green A, Shaddick G, et al. Risk of type 2 diabetes and cardiovascular disease in an incident cohort of people with psoriatic arthritis: a population-based cohort study. Rheumatology 2019;58:144-8.

6. Pearl RL, Wan MT, Takeshita J, et al. Stigmatizing attitudes toward persons with psoriasis among laypersons and medical students. $J$ Am Acad Dermatol 2019;80.

7. Parisi R, Webb RT, Kleyn CE, et al. Psychiatric morbidity and suicidal behaviour in psoriasis: a primary care cohort study. $\mathrm{Br} J$ Dermatol 2019;180.

8. Lee $\mathrm{JH}$, Lee $\mathrm{SM}$, Choi $\mathrm{JH}$, et al. Factors influencing quality of life in patients with psoriasis in Korea. Eur J Dermatol 2018;28:678-680.

9. Michalek IM, Loring B, John SM. A systematic review of worldwide epidemiology of psoriasis. J Eur Acad Dermatol Venereol 2017;31:205-12.

10. Parisi R, Symmons DP, Griffiths CE, et al. Global epidemiology of psoriasis: a systematic review of incidence and prevalence. J Invest Dermatol 2013:133:377-85.

11. Organizaiton WH. Global Report on Psoriasis. Geneva: World Health Organization, 2016.

12. Griffiths CEM, van der Walt JM, Ashcroft DM, et al. The global state of psoriasis disease epidemiology: a workshop report. $\mathrm{Br} J$ Dermatol 2017;177:e4-e7.

13. Shao CG, Zhang GW, Wang GC. Distribution of psoriasis in China: a nationwide screening. Proceedings of the Chinese Academy of Medical Sciences and the Peking Union Medical College. 1987:2:59-65.

14. Li MJ, Wang P, Cai M, et al. Prevalence and Risk Factors of Psoriasis in Hainan Province: an Epidemiological Survey. Chin J Dermatol 2013;46:157-9.

15. Wang RL, Cao LS, Zhou C, et al. Prevalence of 15 Skin Diseases in Adolescents from Liangshan Prefecture in Sichuan Province. Chin J Dermatol 2012;45:270-2.

16. Li R, Sun J, Ren LM, et al. Epidemiology of eight common rheumatic diseases in China: a large-scale cross-sectional survey in Beijing. Rheumatology 2012;51:721-9.

17. Ding $X$, Wang $T$, Shen $Y$, et al. Prevalence of psoriasis in China: a population-based study in six cities. Eur J Dermatol 2012;22:663-7.

18. Xu YY, Tong ZC, Shen SF, et al. An Epidemiological Survey of Psoriasis among rural residents in Suzhou, Anhui Province. Acta Universitatis Medicinalis Anhui 2001;36:483-5.

19. Jin Y. Sampling: Theory and Application. 2nd ed. Beijing: Higher Education Press, 2016:p33-5.

20. Levy PS, Lemeshaw S. Sampling of populations: methods and applications. 4th edn. New York: Wiley, 2008.

21. World Health Organization. Tuberculosis prevalence surveys: a Handbook. Geneva: World Health Organization, 2011.

22. Wang $L$, Zhang $H$, Ruan $Y$, et al. Tuberculosis prevalence in China, 1990-2010; a longitudinal analysis of national survey data. Lancet 2014;383:2057-64.

23. Ren F, Yu X, Dang W, et al. Depressive symptoms in Chinese assembly-line migrant workers: A case study in the shoe-making industry. Asia Pac Psychiatry 2019;11:e12332.

24. National Bureau of Statistics of China. 2017 China Statistical Year Book, 2017. 